\title{
Avaliação e intervenção no desenvolvimento MOtor de UMA CRIANÇa COM SíndRome de DoWn
}

\author{
AsSESSMENT AND INTERVENTION IN THE MOTOR DEVELOPMENT OF A CHILD \\ WITH DOWN SYNDROME
}

\author{
Ana Paula Maurilia dos SANTOS \\ Silvio Luiz Indrusiak WEISS 2 \\ Geciely Munaretto Fogaça de ALMEIDA ${ }^{3}$
}

RESUM O: o objetivo deste estudo foi analisar o desenvolvimento motor de uma criança com síndrome de Down e verificar os efeitos de um programa de intervenção motora específica. Trata-se de uma pesquisa descritiva do tipo estudo de caso. Para a avaliação do desenvolvimento motor foram utilizados os testes da Escala de Desenvolvimento Motor - EDM que analisa as áreas da motricidade fina e global, equilíbrio, esquema corporal, organização espacial e temporal/linguagem, e lateralidade. Essa criança participou, respectivamente, de avaliação motora, intervenção motora (32 sessões, 2 vezes semanais) e reavaliação motora. As intervenções motoras mostraram avanços positivos nas áreas da motricidade global, equilíbrio e organização espacial. A motricidade fina, esquema corporal e a organização temporal / linguagem não apresentaram avanços. Verificou-se que a linguagem foi a área de maior prejuízo. O quociente motor em todos os itens foi classificado como muito inferior, o que se caracteriza como déficit motor. Esses dados justificam a relevância de programas de intervenção motora para essa população.

PALAVRAS-CHAVE: desenvolvimento motor; Síndrome de Down; avaliação motora; intervenção; educação especial.

ABSTRACT: the objective of this study was to analyze the motor development of a child with Down syndrome and to verify the effect of a specific motor intervention program. This is a descriptive research case study. Motor development was evaluated using the Motor Development Scale-MDS, which analyzes both fine and gross motor skills as well as balance, body schema, spatial and temporal organization, language, and laterality. This child participated, respectively, of the motor assessment, motor intervention (32 sessions, twice weekly) and motor reevaluation. Gains were demonstrated in motor intervention in the areas of the gross motor skills, balance and spatial organization. No improvement was shown in fine motor skills, body schema and temporal organization/ language. Language was found to be the area of lowest achievement. The motor quotient for all items was classified as very low, characterizing motor deficit. The data justifies the relevance of motor intervention programs for children with Down syndrome.

KEYWORDS: Motor development; Down Syndrome; Motor Assessment; Intervention; Special Education.

\footnotetext{
${ }^{1}$ Graduada em Educação Física pela Universidade do Estado de Santa Catarina/ UDESC. Aluna especial da disciplina de Desenvolvimento Humano, do curso de M estrado em Ciências do Movimento Humano / UDESC. aninhap@tutopia.com.br

${ }_{2}^{2}$ M estre em N eurociências pela Universidade Federal de Santa Catarina. Professor da Universidade do Estado de Santa Catarina (UDESC/ CEFID) e em programas de Pós-graduação. silvioliw@gmail.com

${ }_{3}^{3}$ Mestre em Ciências do Movimento Humano pela Universidade do Estado de Santa Catarina/ UDESC. Professora da FACVEST (Lages/ SC) nos cursos deFisioterapia, Educação Física ePsicologia. geciely@gmail.com
} 


\section{IINTRODUÇÃo}

Sabe-se, através de diferentes estudos e pesquisas (FONSECA, 1983; WALLON, 1989; ALMEIDA, 2007; ROSA et al., 2008) que crianças com al guma necessidade especial - seja ela física ou mental - apresentam um desenvolvimento mais tardio das funções motoras e cognitivas, o quenão significa quenão venham a atingir um grau de normalidade ou até mesmo superior nos diferentes âmbitos e estágios de desenvolvimento.

Em relação à síndrome de Down, os distúrbios associados podem interferir na aquisição motora dessas crianças, tornando-as muitas vezes vulneráveis ao aprendizado. A hipotonia está altamente correlacionada com atrasos no desenvolvimento, incluindo atrasos no desenvolvimento da motricidade fina eglobal, assim com atrasos em outras áreas do desenvolvimento, como a aquisição da fala e do desenvolvimento cognitivo (TECKLIN, 2002).

Muito embora os indivíduos com síndrome de Down apresentem características peculiares, isso não se constitui numa uniformidade a predizer comportamentos e potencialidades (BISSOTO, 2005). Pode-se dizer que, atualmente, os limites no desenvolvimento das crianças com síndrome de Down não estão firmemente estabelecidos e que vão depender muito diretamente da idoneidade dos programas de estímulos oferecidos a elas. Há necessidade de avaliação e intervenção específica, com equipe multidisciplinar (PACANARO; SANTOS; SUEHIRO, 2008), desde o nascimento (estimulação precoce), passando pela infância e adolescência (estimulação psicomotora) até a vida adulta (estimulação voltada à produção/ trabalho).

Silva e Kleinhans (2006) salientam a necessidade de os programas de intervenções não se limitarem ao atendimento somente de crianças pequenas. De acordo com Almeida et al. (2007), as crianças de zero a três anos geralmente recebem a estimulação precoce em centros especial izados ou APA E's (A ssociação dos Pais e A migos dos Excepcionais) porém, a partir dessa idade, a estimulação reduz consideravelmente ou até mesmo cessa. Luiz et al. (2008) relata o caso da APAE de Ribeirão Preto onde o trabalho da equipe multidisciplinar é mais evidenciado na estimulação precoce, porém, há ofertas de programas voltados às crianças que já foram inseridas na Educação Infantil da rede regular, no entanto, a ênfase está ao atendimento educacional.

Nesse sentido, eleva-se a importância do desenvolvimento motor durante a infância, considerando também que o acompanhamento da aptidão motora de crianças em idade escolar constitui atitude preventiva quanto à aprendizagem, já que estudos que relacionam desenvolvimento motor e rendimento escolar (FONSECA et al., 1994; POETA; ROSA NETO, 2007) demonstram significância estatística entre o que a criança é capaz de aprender (cognitivo) e o que é capaz de fazer (motor). 
Um dos instrumentos muito utilizados na avaliação do desenvolvimento motor é a Escala de Desenvolvimento M otor (EDM), que avalia e acompanha a evolução motora da criança dos 2 aos 11 anos, atribuindo quantitativamente uma idade motora e um quociente motor, determinando as áreas fortes e fracas da motricidade e subsidiando o planejamento do programa de intervenção motora (ROSA NETO, 2002).

Considerando queépossível melhorar a habilidademotora decrianças com farta estimulação psicomotora, o objetivo deste artigo é apresentar o case de avaliação e intervenção motora junto a uma criança com síndrome de Down, discutindo os comportamentos típicos e a intervenção motora adequada, procurando promover conhecimentos e subsídios para os profissionais que atendem crianças com síndrome de Down, em relação ao seu desenvolvimento global e escolar, analisando os resultados do processo de intervenção.

\section{Método}

A pesquisa caracterizou-se como um estudo descritivo, com delineamento de estudo de caso (THOMAS; NELSON, 2002). Este tipo de pesquisa tem como características observar, registrar, analisar e descrever fatos ou fenômenos, procurando descobrir com precisão a frequência em que um fenômeno ocorre e sua relação com outros fatores (MATTOS; ROSSETO JUNIOR; BLECHER, 2004).

\subsection{Participante da Pesquisa}

A criança, do sexo masculino, com 7 anos de idade cronológica, participava do Programa de Estimulação Psicomotora Específica - PIPE, desenvolvido pelo Laboratório de Desenvolvimento Humano, do Centro de Ciências da Saúde e do Esporte - CEFID, da Universidade do Estado de Santa Catarina - UDESC, em Florianópolis/ SC.

Conforme relatado pela mãe, a respeito dos antecedentes pré, peri e pós-natal, a criança nasceu com idade gestacional de 8 meses por parto normal. A presentou, ao nascer, peso de 2.750 gramas com 49 centímetros de altura; notas do A pgar equivalentes a 9 no primeiro minuto, e 8 no 5ㅇminuto. A idade materna no momento do parto era de 27 anos ( 1 o filho).

Quanto ao desenvolvimento neuropsicomotor, a criança sentou sozinha entre os 6 e 12 meses, e começou a caminhar sem apoio aos 2 anos de idade. O sono, desde os primeiros anos de vida, sempre foi tranquilo. Vive em condições sócioeconômicas estáveis, com renda familiar superior a 10 salários mínimos. Sua mãe possui o Ensino Médio completo e exerce a função de Auxiliar Administrativa, e o pai, com o Ensino Médio incompleto, é técnico em manutenção. 


\subsection{Procedimentos e instrumentos de coleta de dados}

O presente estudo é um projeto de iniciação científica subsi diado pelo CNPq. A pesquisa foi aprovada pelo Comitê de Ética em Pesquisa em Seres Humanos da Universidade do Estado de Santa Catarina (UDESC), com protocolo número 130/2005. A partir da autorização dos pais ou responsáveis, através do Termo de Consentimento Livre e Esclarecido, foi realizada a avaliação inicial da criança, cujos instrumentos foram:

a) Questionário biopsicossocial contendo 26 questões fechadas e semiabertas relativas aos dados da criança (data de nascimento, sexo); dados dos pais ou responsáveis (profissão, escolaridade, situação conjugal); condições ligadas à gestação (duração da gravidez, idade da mãe); condições ligadas ao nascimento da criança (tipo de parto, peso, altura, Apgar); desenvolvimento neuropsicomotor (idadeem quesentou ecaminhou sozinha) e condições sócio-econômicas (renda familiar, tipo de moradia).

b) Escala de Desenvolvimento Motor - EDM, de Rosa N eto (2002) para avaliar o desenvolvimento motor, mediante as 7 baterias de testes para motricidade fina e global, equilíbrio, esquema corporal, organização espacial e temporal, bem como a lateralidade. Este instrumento determina a idade motora (obtida através dos pontos alcançados nos testes) eo quociente motor (obtido pela divisão entre a idade cronológica multiplicado por 100). Com exceção dos testes de lateral idade, as outras baterias consistem em 10 tarefas motoras cada, distribuídas entre 2 e 11 anos, organizadas progressivamente em grau de complexidade, sendo atribuído para cada tarefa, em caso de êxito, um valor correspondente à idade motora (IM), expressa em meses. Em cada bateria, o teste é interrompido quando a criança não concluir a tarefa com êxito, conforme protocolo. Ao final da aplicação, dependendo do desempenho individual em cada bateria, é atribuída à criança uma determinada $\mathrm{IM}$, em cada uma das áreas referidas anteriormente (IM 1, IM2, IM 3, IM4, IM 5, IM 6), sendo, após, calculada a idade motora geral (IMG) e o quociente motor geral (QMG) da criança. Esses valores são quantificados e categorizados conforme a Tabela 1.

Tabela 1 - Valores finais do quociente motor e a sua categoria de diagnóstico

\begin{tabular}{cc}
$\begin{array}{c}\text { Quocientes motores em meses } \\
130 \text { ou mais }\end{array}$ & $\begin{array}{c}\text { Desenvolvimento Motor } \\
\text { Muito superior }\end{array}$ \\
\hline $120-129$ & Superior \\
$110-119$ & Normal alto \\
$90-109$ & Normal médio \\
$80-89$ & Normal baixo \\
$70-79$ & Inferior \\
69 ou menos & Muito inferior \\
\hline
\end{tabular}

Fonte: Rosa N eto (2002) 
As etapas do estudo envolveram a seguinte sequência: 10 passo avaliação biopsicossocial / 20 passo - avaliação motora / 30 passo - aplicação do programa de intervenção / 40 passo - reavaliação motora.

\subsection{Programa de intervenção}

As intervenções do Programa de Estimulação Psicomotora Específica - PIPE foram ministradas pelos próprios pesquisadores e ocorreram nas dependências do CEFID/ UDESC, em espaço amplo, iluminado e sem barulhos. O programa desenvolvido teve um total de 32 intervenções, organizadas em duas sessões semanais, com duração de 50 minutos cada.

O objetivo geral do programa de intervenção foi estimular o desenvolvimento da motricidade da criança com síndrome de Down, proporcionando o aprimorando de todas as áreas. As atividades foram realizadas de forma lúdica, e englobaram as áreas da motricidade fina e global, equilíbrio, esquema corporal, organização espacial e temporal, bem como a lateralidade. A criança participou das intervenções motoras com mais três alunos com síndrome de Down, desempenhando as mesmas atividades propostas para os outros al unos da turma. A pós o período estipulado para as sessões de intervenção, a criança foi submetida a uma reavaliação motora com a EDM (ROSA NETO, 2002). O Quadro 1, a seguir, apresenta o programa de intervenção motora, detalhado, utilizado no estudo.

\begin{tabular}{|c|c|c|c|}
\hline $\begin{array}{l}\mathbf{N}^{\circ} \text { de } \\
\text { Sessões }\end{array}$ & $\begin{array}{c}\text { Objetivos } \\
\text { Estimular/desenvolver }\end{array}$ & Conteúdo & Atividades \\
\hline 05 & $\begin{array}{l}\text { Óculo manual e } \\
\text { Destreza }\end{array}$ & Motricidade fina & $\begin{array}{l}\text { Jogos de encaixe, separar blocos por } \\
\text { cores, desenho, pintura, pinçar } \\
\text { pequenos objetos, passar fio na tela, } \\
\text { furar pontos em isopor. }\end{array}$ \\
\hline 05 & $\begin{array}{l}\text { Coordenação e } \\
\text { Controle do corpo }\end{array}$ & Motricidade global & $\begin{array}{l}\text { Circuito com obstáculos (banco, } \\
\text { elástico, corda, bola, cones), jogos } \\
\text { com música (estátua), vivo ou morto, } \\
\text { jogos com bolas. }\end{array}$ \\
\hline 04 & $\begin{array}{l}\text { Postura, Equilíbrio } \\
\text { dinâmico/estático }\end{array}$ & Equilíbrio & $\begin{array}{l}\text { Diferentes posições, com diversos } \\
\text { materiais (auxiliares ou não), jogos de } \\
\text { troca de nível (subir e descer, correr e } \\
\text { parar), etc. }\end{array}$ \\
\hline 04 & $\begin{array}{c}\text { Imitação, Partes do corpo e } \\
\text { Rapidez }\end{array}$ & Esquema corporal & $\begin{array}{l}\text { Brincadeiras no } \\
\text { relacionamentos (monitor, pais, } \\
\text { amigos), brincadeiras cantadas, } \\
\text { atividades com papel, tinta e figuras } \\
\text { geométricas, etc. }\end{array}$ \\
\hline 04 & $\begin{array}{l}\text { Percepção do espaço e } \\
\text { Percepção sensorial }\end{array}$ & Orientação espacial & $\begin{array}{l}\text { Jogos de quebra cabeça e encaixe, } \\
\text { manusear vários materiais e classificar } \\
\text { (grande/pequeno, grosso /fino, } \\
\text { mole/duro). }\end{array}$ \\
\hline 04 & $\begin{array}{l}\text { Linguagem e } \\
\text { Estruturas temporais }\end{array}$ & $\begin{array}{l}\text { Orientação } \\
\text { temporal }\end{array}$ & $\begin{array}{l}\text { Estimular a composição de frases, } \\
\text { conversar, cantar, brincadeiras com } \\
\text { música (ritmo), palmas, bater o pé e } \\
\text { parar. }\end{array}$ \\
\hline 06 & $\begin{array}{l}\text { Dominância de mãos, pés, } \\
\text { olhos }\end{array}$ & Lateralidade & $\begin{array}{l}\text { Jogos de encaixe, arremesso, jogo } \\
\text { com bolas, saci-pererê, circuitos, } \\
\text { máscara, binóculo, olho mágico. }\end{array}$ \\
\hline
\end{tabular}

Quadro 1- Programa de Intervenção M otora para síndrome de Down: 32 sessões. 


\subsection{ANÁLISE DOS DADOS}

Os dados motores foram tabulados no programa informático da Escala de Desenvolvimento M otor - EDM (ROSA NETO, 2002). Para o tratamento dos dados foi utilizada a análise descritiva, onde se pôde comparar os resultados nos dois momentos deavaliação (prée pós-teste) nas variáveis IdadeCronológica (IC); I Iade e Quociente M otor Geral (IMG e QM G) ; Idades eQuocientes M otores (IM e QM) específico para cada área motora; e Idade N egativa (IN), diferença entre a idade motora geral e idade cronológica , cal culada por meio da subtração $I M G-I C=I N$.

\section{Resultados}

Os resultados abaixo apresentam, inicialmente, a análise descritiva das variáveis de Idade Cronológica (IC), I dade N egativa (IN ), I dade M otora Geral (IMG) e Quociente Motor Geral (QMG) nos dois momentos, pré e pós-teste.

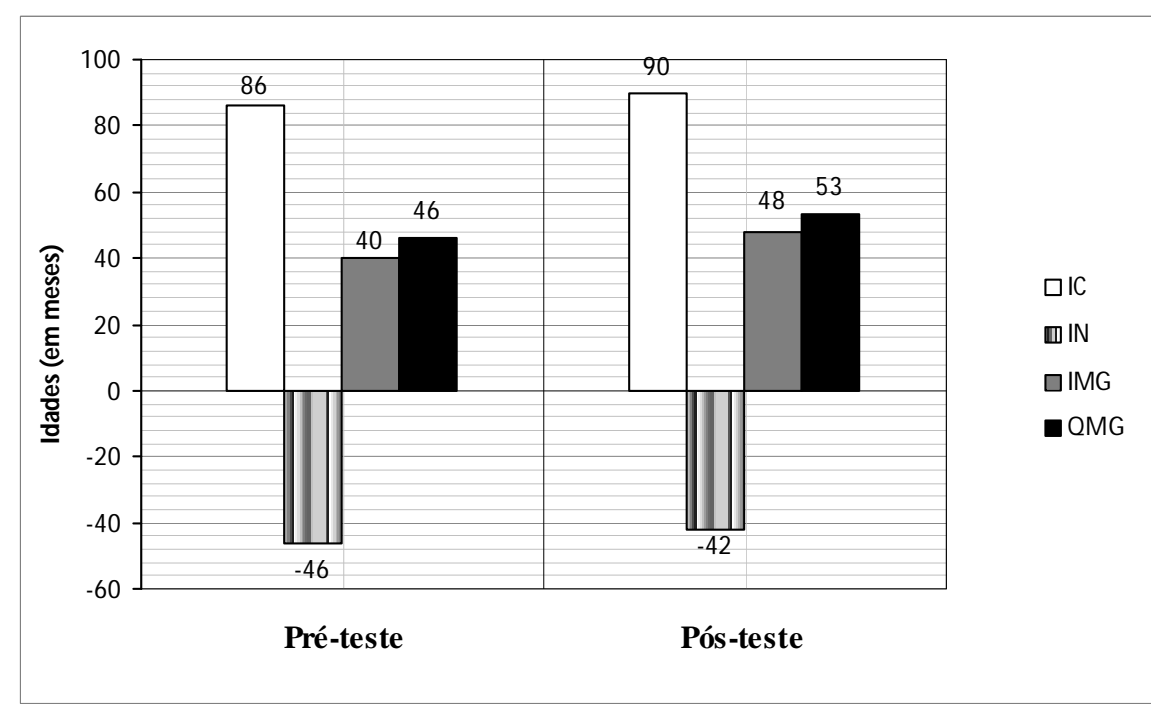

Figura 1 - Idade cronológica (IC), idade negativa (IN), idade motora geral (IMG) e Quociente motor geral (QMG) no pré e pós-teste.

Com relação aos resultados obtidos na Figura 1, a IC da criança foi de 86 meses no préteste e 90 meses no pós-teste - aumento de quatro meses decorrente do período entre a aplicação do pré e pós-teste em que ocorreram as intervenções. A respeito daIN, demonstrou alteração nos seus val ores, que passou de -46 meses para - 42 no pós-teste. Com o aumento da IC, a IN também tende a 
aumentar, no entanto os ganhos na IMG (aumento de oito meses do pré para o pós-teste) favoreceram a diminuição dessa idade negativa - fator positivo para o desenvolvimento da criança. Através do cálculo do QMG pode-se reafirmar o atraso no desenvolvimento motor apresentado por essa criança, que foi classificado como "muito inferior" nos dois momentos (pré e pós-teste).

$\mathrm{Na}$ Figura 2, podese observar as idades motoras específicas antes e após 4 meses de intervenções motoras.

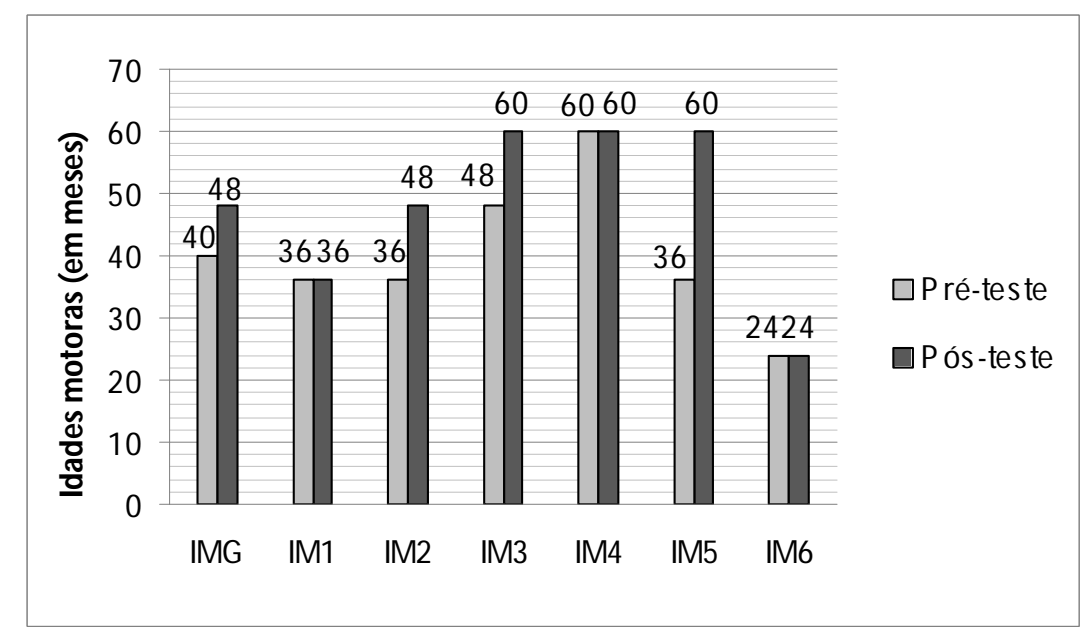

Figura 2 - Distribuição das idades motoras antes e após as intervenções.

$\mathrm{Na}$ análise do desenvolvimento motor da criança, em cada tarefa, pode-se observar um déficit motor em todas as áreas, com maiores prejuízos na organização temporal/ linguagem (IM6) e motricidade fina (IM1). Nas áreas da motricidade global (IM2), equilíbrio (IM3) e organização espacial (IM5) houve melhorias importantes após as intervenções. No esquema corporal (IM4) não ocorreram alterações, assim como na motricidade fina. A lateralidade, que não consta no gráfico, foi definida como destro-completo nos dois momentos.

\section{Dıscussão}

De modo geral, apesar dos baixos resultados motores atingidos pelas crianças com síndrome de Down, programas deestimulação precoceeintervenção são capazes de fazêlas atingir níveis motores mais satisfatórios (LORENZINI, 2002; SÁ N CHES et al., 2003). Esta pesquisa conseguiu comprovar essa afirmativa, pois a criança participante das intervenções psicomotoras obteve resultados positivos, com aumento da IMG e do QMG. Almeida (2006) também encontrou avanços positivos na idade e no quociente motor geral em uma criança 
participante de um programa de intervenção. Côrrea (2005) demonstrou que a aplicação de um programa específico de desenvolvimento motor, composto por atividades lúdicas, beneficiou significativamente as crianças com síndrome de Down. De tal modo, a análise do nosso estudo verificou aumento significativo nas principais áreas motoras da criança.

No equilíbrio, a idade motora da criança evolui de 48 para 60 meses após as intervenções motoras. Outros autores (SILVA; FERREIRA, 2001; GUÉRIOS; GOMES, 2005) também encontraram avanços significativos nessa área em crianças que participavam de um programa de atividades motoras. Conforme Sherril (apud FRANÇA; ZUCHETTO, 2004), muitas crianças com síndrome de Down não conseguem manter o equilíbrio sobre um pé só por mais de dois segundos. A atual pesquisa confirma essa teoria, uma vez que a criança não conseguiu realizar o equilíbrio num pé só - tarefa referida no item 6 anos, da prova de equilíbrio, da EDM (ROSA NETO, 2002). Uma criança com a mesma idade do menino desta pesquisa ( 7 anos) estaria apta a realizar essa tarefa, uma vez que a escala é gradual; no entanto, as características inerentes da síndrome de Down o impede de acompanhar essa sequencia padrão.

Na motricidade global, o QMG foi de 36 meses no pré-teste, passando para 48 meses no pós-teste. Ferreira (apud ROSA, 2008) também encontrou melhorias na motricidade global em crianças com síndrome de Down participantes de um programa de intervenção motora. Spano et al. (apud FUNAYAMA, 2002) realizaram estudos voltados à competência motora em crianças com síndrome de Down e encontraram dados convergentes à atual pesquisa: aplicando testes de competência motora e visuo-motora em crianças com síndrome de Down em idade escolar, observaram aquisições regulares nas atividades motoras grosseiras e grande variabilidade nos movimentos finos - em função do tipo de tarefa, com maiores prejuízos na coordenação bimanual. Quanto aos movimentos finos, vale ressaltar que a sua execução envolve precisão, atenção ehabilidades sensoriais e perceptuais, atributos que estão comprometidos nas crianças com síndrome de Down (GIMENEZ et al., 2004).

No participante desse estudo, também foi verificado um déficit motor considerável na motricidade fina, sendo a segunda área com mais atraso, que apresentou uma idade motora fina (pré e pós-teste) equivalente a uma criança de três anos. Clausen (apud RAMALHO, 2000) considera que a aprendizagem inicial dos padrões de movimentos finos das crianças com síndrome de Down ocorre de maneira lenta e dificultosa. Na pesquisa realizada por esse mesmo autor, as crianças sindrômicas mostraram um pior desempenho na motricidade fina, quando comparada a um grupo de crianças não sindrômicas.

Muitos autores afirmam que a área de desenvolvimento mais comprometida nas crianças com síndrome de Down é a da linguagem, sendo mais evidentes as dificuldades gramaticais e sintáticas. (TRISTÃO; FEITOSA, 1998; DODD; ASSUN ÇÃO, apud RAMALHO, 2000). Estas informações 
corroboram com a criança desta pesquisa, onde a linguagem mostrou-se com maiores prejuízos. Entre outros fatores, uma das justificativas para os resultados abaixo da normalidade é a influência da hipotonia (presente na grande maioria das crianças com síndrome de Down) que interfere nas habilidades linguísticas, prejudicando o controle dos músculos da língua, dificultando a formação de palavras (STRAY-GUNDERSEN, 2007). Para Nichols et al., (2004) a associação da síndrome de Down com anormalidade do desenvolvimento cerebral é um dos motivos da assincronia de desenvolvimento entre a linguagem compreensiva e expressiva. Iverson, Longbardi e Caselli (2003) salientam que a linguagem compreensiva desenvolve-se melhor que a expressiva nessas crianças. Acerca disso, Andrade e Limongi (2007) afirmam que para compensar o atraso de sua produção oral, muitas crianças com síndrome de Down desenvolvem de modo significativo a comunicação gestual, variando-a conformeo contexto do ambiente, com o objetivo de sefazerem mel hor compreendidas pelo interlocutor. Essa teoria, de certa forma, concorda com os dados da atual pesquisa, uma vez que era predominante nas intervenções o uso de gestos, expressões faciais e corporais pela criança. Essa comunicação não verbal era facilmente interpretável, e essa habilidade já era de se esperar, uma vez que na avaliação motora o esquema corporal foi a área em que a criança apresentou maior idade motora. Esse fato, no entanto, não invalida o atraso motor que a criança apresenta no esquema corporal, como também vale ressaltar que não foram observadas melhorias após as intervenções, apresentando idade motora negativa de 30 meses nessa área. Côrrea, Silva e Gesualdo (2005) avaliaram 15 crianças com síndrome de Down (entre 6 e 15 anos) e todas apresentaram também atraso no esquema e na imagem corporal.

Nieves (2007) afirma que o esquema corporal apoia o desenvolvimento de outras áreas motoras tais como a organização espacial etemporal; desta forma, deve-se obter uma perfeita concordância entre essas áreas. Na atual pesquisa, a organização espacial apresentou um aumento expressivo: a idade motora passou de 36 meses para 60 meses após as intervenções - idade motora análoga ao esquema corporal. Em relação às estruturas temporais, percebeu-se o forte comprometimento que a criança apresentou nessa área, com atrasos relacionados às noções de ritmo, noções de tempo e principalmente na linguagem - já tratada anteriormente. Souza (1997), assim como na atual pesquisa, encontrou dificuldades na estruturação espaço-temporal e problemas na verbalização ao analisar o perfil motor de crianças com deficiência mental, e a organização temporal foi também a área de maior déficit nessas crianças.

No que se refere ao desenvolvimento da lateralidade, os dados desta pesquisa indicaram preferência lateral direita, sendo caracterizada como destra completa (mãos, ol hos, pés). Almeida (2007), ao avaliar o desenvolvimento motor de crianças com deficiência mental, encontrou em 32,2\% dessas, dominância lateral destro-completa, no entanto, a grande parte de sua amostra apresentou lateralidade indefinida. Para Lorenzini (2002), crianças com necessidades 
especiais tendem a apresentar al terações na lateral idade, em função das alterações percepto-cognitivas.

\section{CONCLUSÃo}

Levando em conta o objetivo do estudo, foi possível perceber, através dos resultados encontrados, que as áreas do desenvolvimento motor da criança em estudo apresentaram al terações, devi do às limitações inerentes desta síndrome. A Escala de Desenvolvimento Motor - EDM (Rosa N eto, 2002) detectou e classificou os déficits motores da criança como muito inferior nos dois momentos (pré e pós-teste).

Demodo geral, as áreas que apresentaram maiores dificuldades foram a motricidade fina ea linguagem. Uma das justificativas para os baixos resultados encontrados nessas áreas é a forte influência da hipotonia, que interfere nas habilidades linguísticas, prejudicando o controle dos músculos da língua, dificultand o a formação de pal avras; eos atributos necessários à real ização motora fina como precisão, atenção e habilidades sensoriais e perceptuais, que estão comprometidos nas crianças com síndromede Down. No entanto, as intervenções psicomotoras realizadas neste período foram capazes de alterar positivamente a linha de desenvolvimento da criança deste estudo, demonstrando ganhos importantes em seu desenvolvimento global (IMG e QMG) e, principalmente, nas áreas da motricidade global, equilíbrio e organização espacial.

A exemplo das sessões de intervenções psicomotoras em crianças com síndrome de Down, alguns aspectos devem ser levados em consideração. As explicações teóricas dos exercícios devem ser curtas e simples, contendo uma orientação de cada vez, pois nota-se que algumas crianças têm dificuldade de seguir orientações, ou de entender falas um pouco mais extensas. Utilizar a fantasia com essas crianças facilita a vivência dos exerć́cios, assim como amplia a percepção de mundo, estimulando o desenvolvimento do pensamento, integrando-os aos aspectos afetivo-motores. Deve-se dar ênfase aos objetivos das atividades, focando-se, também, nas linhas de vivência como vitalidade, integração, criatividade e afetividade. Às vezes el es apresentam instabilidade emocional, ficam "teimosos", "irritados", "emburrados", negam-se a realizar determinadas atividades. N estas situações o coordenador deveser bastanteflexível e criar uma forma diferente de chamá-los a realizar as atividades, que não seja a insistência. A atual pesquisa conseguiu cumprir seus objetivos, abrindo os horizontes para o assunto do desenvolvimento motor em crianças com síndrome de Down. 


\section{Referências}

ALMEIDA, G. M . F. D eficiên cia mental: avaliação eclassificação do desenvol vimento motor. 2007. 129f. Dissertação (Mestrado em Ciências do M ovimento Humano) - Centro deCiências da Saúdee do Esporte, Universidade do Estado de Santa Catarina, Florianópolis, 2007.

ALMEIDA, G. M. F. et al. PIPE - PROGRAMA DE INTERVENÇÃO PSICOMOTORA ESPECÍFICA. R evista I beroamericana de Psicomotricidad y Técnicas Corporal es. Montevidéu, v. 7, p. 131-136, 2007.

ALMEIDA, G.M.F.; ROSA NETO, F. M otor evaluation and intervention in Down syndrome. FIEP BU LLETIN . Foz do Iguaçu, v.76, Special Edition , p.413-416, 2006.

ANDRADE, R. V; LIMONGI, S. C. O. A emergência da comunicação expressiva na criança com síndrome de Down. Pró-F ono R evista deatual ização científica. São Paulo, v.19, n.4, p. 387392, 2007. Disponível em: 〈http:/ / www.sciel o.br>. A cesso em: 14 mar. 2009.

BISSOTO, M. L. O desenvolvimento cognitivo eo processo deaprendizagem do portador de síndrome de Down: revendo concepções e perspectivas educacionais. Ciências e Cognição; Rio deJaneiro, v.4, n.2, 2005. Disponível em: 〈http:/ / .cienciasecognicao.org/ >. A cesso em: 10 mar. 2009.

CORRÊA, F.I; SILVA, F.P; GESUALDO, T. A valiação da imagem e esquema corporal em crianças portadoras da síndrome de Down e crianças sem comprometimento neurológico. Fisioterapia Brasil, São Paulo, v.4, n.1, p. 19-23, fev 2005.

FRANÇA , C; ZUCHETTO, A.T. Comportamento social deportadores desíndromededown em contexto de atividade motora adaptada. R evista da soci edade brasileira de atividade motora adaptada - sobama, São Paulo, v.9, n.1, dez 2004.

FON SECA, V. P sicomotricidade. São Paulo: Martins Fontes, 1983.

FUNAYAMA, C.A.R. Aspectos neurológicos da síndrome de Down. Temas sobre D esenvolvimento, São Paulo, v.11, n.61, p.40-44, 2002.

GIMENEZ, R; MANOEL E.D; OLIVEIRA, D.L, BASSO, D.L. Combinação de padrões fundamentais de movimento: crianças normais, adultos normais e adultos portadores da síndrome de Down. Revista Paulista de Educação Física, São Paulo, v. 18, p. 101-116, 2004. Disponível em: http:/ / www.usp.br/ eef/ rbefe/ v18n12004/ v18p101 .pdf>Acesso em: 13 mar. 2009.

GUERIOS, L.C; GOMES, N.M. A nálise deum programa para desenvolvimento dos padrões fundamentais demovimento em crianças portadoras desíndrome de down. R evista D igital, Buenos Aires. v.10, n. 83. 2005. Disponível em: বhttp:/ / www. efdeportes.com >. A cesso em: 6 jan. 2009.

IVERSON , J.M; LON GBA RDI, E; CA SELLI, M.C. Relationship between gestures and words in children with Down's syndrome and typically developing children in the early stges of communicative development. Int. J. Lang. Communication D isorders, v.2, n.38; p.179-97, 2003.

LORENZINI, Marlene. Brincando a brincadeira com a criança deficiente. São Paulo: Manole, 2002.

LUIZ, F.M.R et al. A inclusão da criança com síndrome de Down na rede regular de ensino:desafios e possibilidades. Rev. Bras. Ed. Esp., Marília, v.14, n.3, p.497-508, 2008. Disponível em: বhttp:/ / www.scielo.br/ rbee >. A cesso em: 30 fev. 2009. 
MATTOS, M.G; ROSSETTO JÚNIOR, A.J; BLECHER, S. Teoria e prática da metodologia da pesquisa em educação física: construindo sua monografia, artigo científico e projeto de ação. São Paulo: Phorte, 2003.

NICHOLS, S. et al. Mechanisms of verbal memory impairment in four neurodevolopmental disorders. Brain and Language, v. 88, p. 180-189, 2004.

NIEVES, Y.F. Algunas consideraciones sobre psicomotricidad y las necesi dades educativas especiales (NEE). R evista D igital, Buenos Aires, v.12, n.108, 2007. Disponível em: <http:/ / www.efdeportes.com/ >A cesso em: 4 fev. 2009.

PACANARO, S.V; SANTOS, A.A.A; SUEHIRO, A.C.B. A valiação das habilidades cognitiva e Visuo-motora em pessoas com síndrome de Down. Rev. Bras. Ed. Esp., Marília, v.14, n.2, p.293-310, 2008. Disponível em: 〈http:/ / www.scielo.br/ rbee>. A cesso em 05 mai. 2009.

POETA, L; ROSA NETO, F. Evaluación motora en escolares con indicadores del trastorno por déficit de atención/ hiperactividad. R evista de N eurología, v. 44, p. 1112-1115, 2007.

RAMALHO, C.M.J; PEDROM ÔNICO, M.R; PERISSINO, O.J. SíndromedeDown: avaliação do desempenho motor, coordenação e linguagem (entre dois e cinco anos). Temas sobre desenvolvimento. São Paulo, v.9, n.52, p.11-14, 2000.

ROSA NETO, F. M anual de avaliação motora. Porto A legre: Artmed, 2002.

ROSA, G.K.B et al. Desenvolvimento motor de criança com paralisia cerebral: avaliação e intervenção. R ev. Bras. Ed. Esp. v.14, n.2, p.163-176, 2008. http:/ / www.scielo.br/ rbee >. Acesso em: 05 mai. 2009.

SÁNCHES, P.A. et al. A psicomotricidade na educação infantil - uma prática preventiva e educativa. Trad.: Inajara H aubert Rodrigues. Porto A legre: A rtmed, 2003.

SILVA, D.R; FERREIRA, J.S. Intervenções na educação física em crianças com síndromede Down. R evista da E ducação Física /U E M . Maringá, v.12, n.1,p.69-76, 2001.

SILVA, M. F. M. C.; KLEIN HANS, A. C. S. Processos cognitivos e plasticidade cerebral na Síndrome de Down. Rev. Bras. Ed. Esp., v.12, n.1, p.123-138, 2006. Disponível em: বhttp:/ / www.scielo.br/ rbee>. A cesso em: 11 abr. 2009.

SOUSA, D. G. Perfil motor em portadores de deficiência mental em Florianópolis - SC. 1997. Monografia (Graduação em Educação Física) - Centro de Ciências da Saúde e do Esporte, Universidade do Estado de Santa Catarina, Florianópolis, SC, 1997.

STRAY-GUNDERSEN. Crianças com Síndrome de D own: guia para pais e educadores. Porto Alegre: Artmed, 2007.

TECKLIN , J.S. Fisioterapia pediátrica. 3.ed. Porto A legre: A rtmed, 2002.

THOMAS, J. R.; NELSON , J. K. M étodos de pesquisa em educação física. Porto A legre: Artmed, 2002.

TRISTÃO, R. M; FEITOSA, M.A. Linguagem naSíndromedeDown. Psicologia: Teoria eP esquisa, Brasília, v. 14, n. 2, p. 127-137, 1998.

WA LLON, Henri. A s origens do pensamento na criança. São Paulo: Manole, 1989.

Recebido: 29/ 05/ 2009

Reformulado: 11/ 01/ 2010

A provado: 12/ 02/ 2010 